\title{
Spectral and Energy Morphology Analysis Study of HAWC J2031+415
}

\section{lan Herzog ${ }^{a, *}$ on behalf of the HAWC Collaboration}

(a complete list of authors can be found at the end of the proceedings)

a Michigan Technological University,

1400 Townsend Drive, Houghton, USA

E-mail: igherzog@mtu.edu

The Cygnus Cocoon region is a complex region containing an OB star cluster that is prominent in

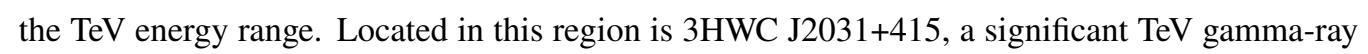
source whose emission is possibly associated with 2 components, the Cygnus OB2 star cluster and a pulsar wind nebula (PWN). In this work, several modelling methods are presented to best describe the emission. These models disentangle emission believed to be from the Cocoon and isolate the component emitted by the probable PWN. I will present several spectral models to describe the emission of the probable PWN using the latest data set from the High-Altitude Water Cherenkov (HAWC) observatory. Furthermore, I will present an energy morphology study of the PWN component of 3HWC J2031+415 in distinct energy bands.

$37^{\text {th }}$ International Cosmic Ray Conference (ICRC 2021)

July 12 th - 23rd, 2021

Online - Berlin, Germany

\footnotetext{
${ }^{*}$ Presenter
} 


\section{Introduction}

The first detection of a TeV source close to 3HWC J2031+415 was by HEGRA in 2002 and it was unique in that it had no obvious counterpart in lower energy ranges [7]. Designated TeV $\mathrm{J} 2032+4130$, they performed a point source fit to its emission and placed it firmly in the Cygnus Cocoon region.

The first detection of a TeV source close to 3HWC J2031+415 was TeV J2032+4130, an extended very high energy source detected by HEGRA in 2002 [7]. Located in the Cygnus Cocoon region (henceforth referred to as the Cocoon) in the constellation Cygnus, it was unique in that there was no obvious counterpart in lower energy ranges. A later study by VERITAS resulted in an extended asymmetric Gaussian fit being determined with data between .5 to $30 \mathrm{TeV}$ [6]. Furthermore, they hypothesized that the PWN is powered by PRS J2032+4127, a binary pulsar located near the source of the emission [2,6]. As a result of this hypothesis, they predicted a cutoff in the spectrum at a few tens of $\mathrm{TeV}[6]$.

The latest work on $\mathrm{TeV} \mathbf{J} 2032+4130$ comes from the HAWC observatory in both the 3rd HAWC catalog, which gave it the name 3HWC J2031+415, and a dedicated analysis on the Cocoon region, calling it HAWC J2031+415 [1, 4]. The publication on the Cocoon resolved 3HWC J2031+415 into two distinct sources: the TeV continuation of the Cygnus Cocoon (HAWC J2030+409) and HAWC J2031+415 the PWN previously detected by VERITAS [4]. That study focused on isolating the Cocoon emission and as such they modelled the PWN and subtracted it out of the region. This work will explore HAWC J2031+415's emission and resolve its energy morphology.

\section{Instrument and analysis}

The HAWC observatory is a wide field of view TeV gamma-ray observatory located at Sierra Negra in Mexico at an elevation of 4100 meters [3]. The main array consists of 300 water Cherenkov detectors that covers approximately $22,000 \mathrm{~m}^{2}$ and is sensitive to gamma-rays in the range of 300 $\mathrm{GeV}-100 \mathrm{TeV}$ and beyond [1,3]. A secondary array called the outriggers is currently being implemented and contains an additional 345 smaller tanks and increasing the effective area to $100,000 \mathrm{~m}^{2}$. Each main array tank contains 4 photomultiplier tubes (PMT) that capture Cherenkov light emitted by high energy particles entering the tanks. These events are collected and background and data maps are constructed from them $[1,3]$.

HAWC data is binned using 3 methods: fhit, ground parameter, and the neural network $[1,3]$. The fhit binning method (bins 1-9) takes the percent of PMT's triggered by a shower and is efficient at low energies; at above $10 \mathrm{TeV}$ much of the sensitivity is lost as the whole array is triggered. A further refinement that was developed was the "ground parameter" energy estimator. This method divides each fhit bin into 12 quarter decade bins (a-l) and results in much higher sensitivity above 10 $\mathrm{TeV}$. The reconstruction method takes the charge density 40 meters from the shower axis to determine various binning parameters [3]. The neural network method trains a network to reconstruct the shower using a variety of parameters and is not considered for this analysis. This study will use 1343 days of ground parameter data with reconstructed energies $>1 \mathrm{TeV}$. 


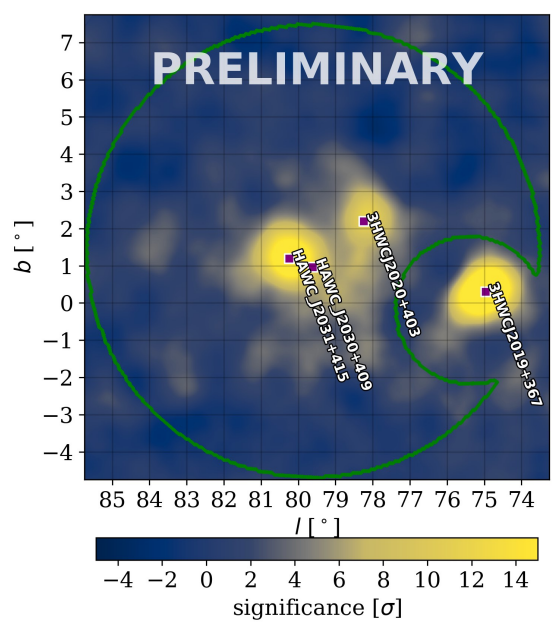

(a) Significance map of the Cocoon region. This shows the excess before removing 3HWC J2020+403 and HAWC J2030+409.

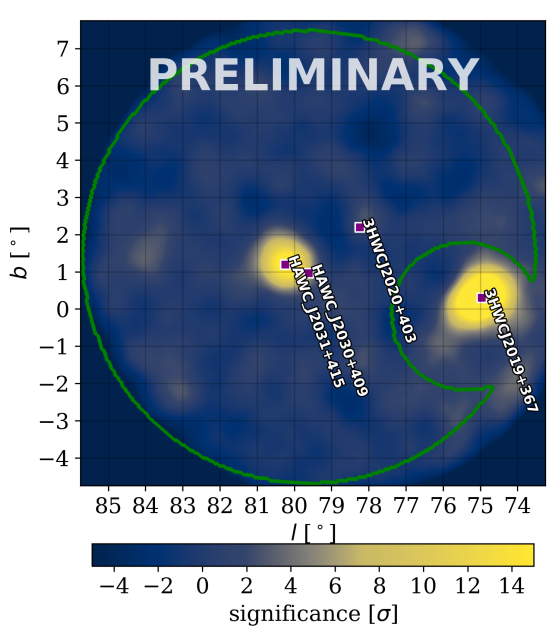

(b) Significance map after subtracting 3HWC J2020+403 and HAWC J2030+409 as discussed in Section 3.1. There is a significant excess after subtraction.

Figure 1: Significance maps of the Cocoon region. Both maps are made with a $0.5^{\circ}$ extension and index of -2.7 assumptions and use 1343 days of ground parameter HAWC data with reconstructed energy $>1 \mathrm{TeV}$. The green contour shows the ROI considered.

\begin{tabular}{|c|c|c|c|c|}
\hline Source & R.A [deg] & Dec. [deg] & Morphology & Source Association \\
\hline $3 \mathrm{HWC}$ J2020+403 & 305.27 & 40.50 & 0.63 (fixed) ${ }^{\circ}$ Gaussian width & Gamma Cygni SNR \\
\hline HAWC J2030+409 & 307.65 & 40.93 & $2.18 \pm 0.17^{\circ}$ Gaussian width & Cygnus Cocoon \\
\hline HAWC J2031+415 & 307.82 & 41.51 & $0.27 \pm 0.02^{\circ}$ Gaussian Width & VER J2031+415 PWN \\
\hline
\end{tabular}

Table 1: The sources considered with location, morphology, and their associated counterparts [4, 6]

\section{Modelling the region}

Shown in Figure 1a is the initial significance map of the region of interest (ROI) considered for this analysis. 3HWC J2031+415 is the second brightest source in the Cocoon region and is detected with a significance of $23.6 \sigma$ at the location $\mathrm{RA}=307.93^{\circ}$, Dec $=41.51^{\circ}$ [1]. As previously mentioned, 3HWC J2031+415 can be resolved as HAWC J2030+409 and HAWC J2031+415. Furthermore an additional source, 3HWC J2020+403, also known as the Gamma Cygni supernova remnant, will also be considered for this analysis [12]. The locations, morphologies, and the nearest associated sources are given in Table 1. The ROI is a 6 degree circle centered at RA=306.5 , Dec $=40.7^{\circ}$, with a 2 degree mask placed on the brightest source in the region, 3HWC J2019+367, and is shown in Figure 1a. 


\subsection{Fitting the region}

A multi-source likelihood fitting method is incorporated using the HAL ${ }^{1}$ and $3 \mathrm{ML}$ framework [11]. All 3 sources are modelled as extended Gaussians and are initially fitted simultaneously. Both 3HWC J2020+403 and HAWC J2030+409 are simple power laws (PL) while HAWC J2031+415 is verified as a power law with an exponential cutoff (PLC) as predicted by VERITAS $[4,6]$. The simple power law equation is shown in Equation 1 and the power law with an exponential cutoff form is given in Equation 2.

$$
\begin{gathered}
\frac{d E}{d N}=N_{o}\left(\frac{E}{E_{p}}\right)^{-\gamma} \\
\frac{d E}{d N}=N_{o}\left(\frac{E}{E_{p}}\right)^{-\gamma} * \exp \frac{-E}{E_{c}}
\end{gathered}
$$

The free parameters are as follows: 3HWC J2020+403 has flux $\left(N_{o}\right)$ and index $(\gamma)$, HAWC J2030+409 has flux $\left(N_{o}\right)$, index $(\gamma)$, and Gaussian width $(\sigma)$, and HAWC J2031+415 has flux $\left(N_{o}\right)$, index $(\gamma), \sigma$, and the cutoff $\left(E_{c}\right)[4,12]$. The pivot energies $\left(E_{p}\right)$ are $1.1 \mathrm{TeV}$ for $3 \mathrm{HWC} \mathrm{J} 2020+403$, 4.2 TeV HAWC J2030+409, and 4.9 TeV for HAWC J2031+415 [4]. Once the fit is completed, the residual map displays no significant emission.

To isolate HAWC J2031+415, the best fit parameters found for the Cocoon and Gamma Cygni are then fixed in the next iteration of the fitting process. This is to subtract out their emission and leave only HAWC J2031+415, and this is shown in Figure 1b. HAWC J2031+415 is then fitted again and compared to the 3 source fitting results to verify the validity of the subtraction method.

\section{Morphology study}

Now that HAWC J2031+415 has been properly modelled, an investigation of its energy morphology is done. It has been shown that the HAWC array does not have a large enough data set or adequate angular resolution to perform well constrained fits with individual bins or energy bands for bright sources as found in these systematic studies [5,13]. As such, a slicing method is considered. Broadly speaking, this method takes a rectangular region of data and slices into a specified number of bins and counts the excess events. Each slice effectively creates a smaller rectangle inside the larger rectangular region considered. For this analysis, 50 excess event bins are considered to maximize the bin number while avoiding pixelation issues that can arise from slicing HEALPIX pixels too finely $[5,13]$. These bins will henceforth be referred to as count bins. These count bins are then plotted in longitudinal profiles and a 1D Gaussian is fitted to the trend the count bins trace. The Gaussian width is then treated as the width and is defined as PSF' for the 68\% containment $\sigma$. This $\sigma$ is approximately the width of the source, though comparison to simulations is required [5,13].

For HAWC J2031+415, the data set considered is the subtracted set as referenced in Section 3.1. To maximize the data included in the energy bands, 4 bands will be used with the following reconstructed energy ranges: Band $1(1-3.16 \mathrm{TeV})$, Band $2(3.16-10 \mathrm{TeV})$, Band $3(10-56.2$ $\mathrm{TeV}$ ), and Band 4 (56.2 -316 TeV). These correspond to ground parameter bins $\mathrm{c} \mathrm{d}$, e f, $\mathrm{g} \mathrm{h}$ i, and

\footnotetext{
${ }^{1}$ https://github.com/threeML/hawc ${ }_{h}$ al
} 
$\mathrm{j} \mathrm{k} 1$ respectively [3]. As has been previously found, not all the bins have enough data due to the energy binning scheme and corresponding PSF' and so a combination of point source simulations and data comparisons must be done to reduce the total bin number to maximize data and minimize PSF' $[3,13]$. To do this, both the Crab Nebula and HAWC J2031+415 are simulated as simple PL and point sources at their respective known locations. This is to also observe whether there is a significant difference with PSF' and declination. The results are shown in Figure 2.

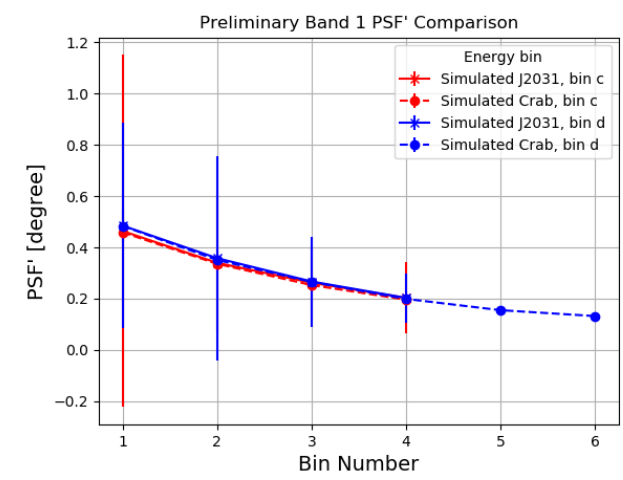

(a) Band $1(1-3.16 \mathrm{TeV})$. This band contains all energy bins $\mathrm{c}$ and $\mathrm{d}$.

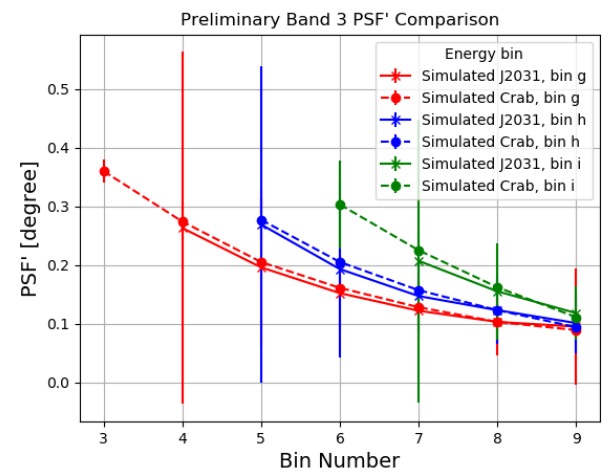

(c) Band 3 (10 -56.2 TeV). This band contains energy bins $\mathrm{g}, \mathrm{h}$, and $\mathrm{i}$.

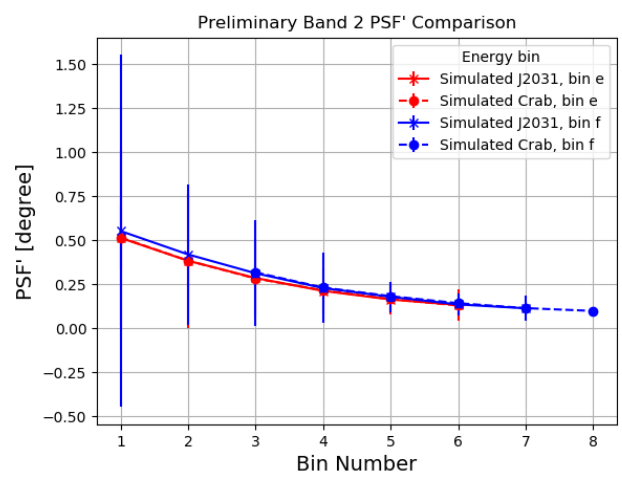

(b) Band 2 (3.16-10 TeV). This band contains energy bins e and $\mathrm{f}$.

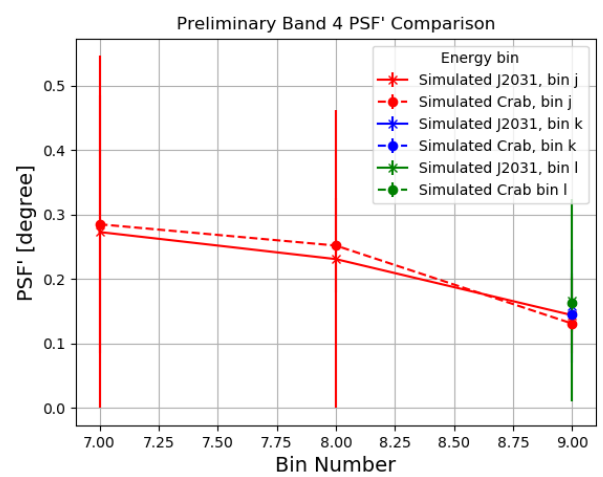

(d) Band 4 (56.2 - $316 \mathrm{TeV})$. This band contains energy bins $\mathrm{j}, \mathrm{k}$, and $\mathrm{l}$.

Figure 2: Comparison of PSF' of HAWC J2031+415 and Crab simulations. The $x$-axis represents each fhit bin that has a corresponding ground parameter energy bin (1c, 2c, etc). The y-axis is the PSF' of each count bin. As it can be see, the simulated PSF' of both sources very closely match and can be approximated as equivalent.

It can be seen that there is no significant declination dependence with PSF' and as such the simulated Crab bins will be treated to determine the best count bins for each band. The combined energy bin bands can be seen in Figure 3. Here all energy bins corresponding to each fhit bin are combined. The signal/noise $(\mathrm{S} / \mathrm{N})$ ratio is determined by dividing excess counts by the background.

The bins kept correspond to the highest $\mathrm{S} / \mathrm{N}$ value and any that follow that bin, if applicable. If bins with lower S/N values are kept, the PSF' should be roughly $75 \%$ of the highest S/N PSF', though exceptions can be made. For example, Band 1 contains bins 3 and 4 . Bin 5 is not included as there is insufficient data at HAWC J2031+415's location. With these bands a proper morphology study 


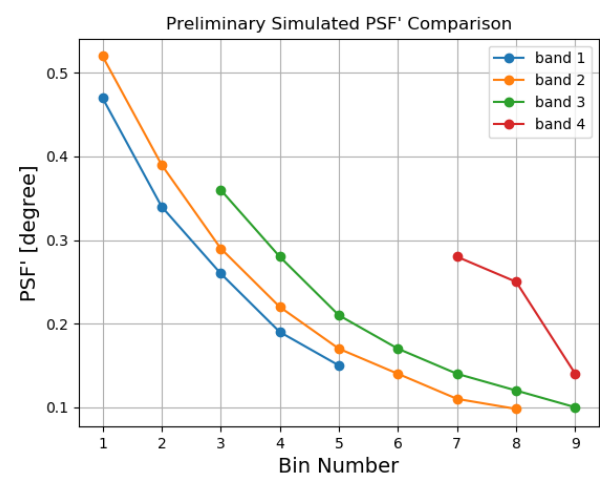

(a) Comparing PSF' of each combined fhit bin of each energy band

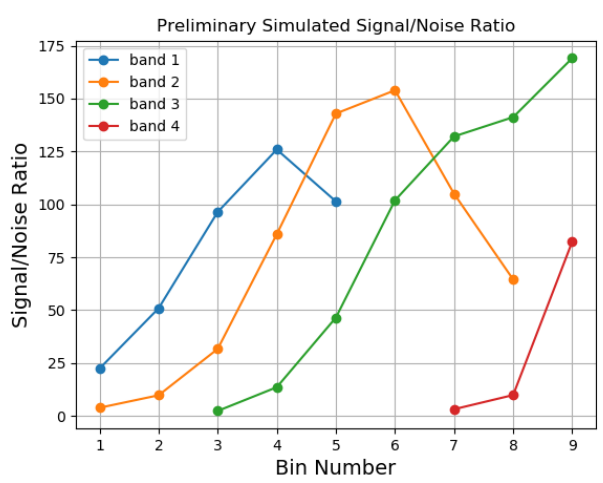

(b) Comparing the $\mathrm{S} / \mathrm{N}$ ratio of each combined fhit bin of each energy band.

Figure 3: Determining the best bins to keep for each energy band. Each band is color-coded and corresponds to their respective energy intervals.

can be conducted. The results are inconclusive, there is no significant trend towards decreasing energy morphology with increasing energy bands of HAWC J2031+415.

\section{Discussion and conclusions}

This is the first in-depth high energy analysis of HAWC J2031+415. The morphology of this source agrees with previous work within error bars and is detected in the energy spectrum to 100 $\mathrm{TeV}$ [4]. Considering the morphology study, issues were encountered with the amount of statistics contained in each band. As the energy increased, there became too little data to adequately constrain the $\sigma$ of the fit. However, this will be addressed soon as the outrigger array addressed earlier is being brought online. This array will significantly increase HAWC's sensitivity of $>10 \mathrm{TeV}$ events. This will greatly aid with this morphology study and may reveal additional shifts not currently visible. Additionally, further work from the LHAASO observatory could reveal additional structure to this region. With the current data set, HAWC J2031+415 is not visible above $100 \mathrm{TeV}$ but the high energy detection by LHAASO in this region will be considered in future work [14].

\section{Acknowledgments}

We acknowledge the support from: the US National Science Foundation (NSF); the US Department of Energy Office of High-Energy Physics; the Laboratory Directed Research and Development (LDRD) program of Los Alamos National Laboratory; Consejo Nacional de Ciencia y Tecnología (CONACyT), México, grants 271051, 232656, 260378, 179588, 254964, 258865, 243290, 132197 , A1-S-46288, A1-S-22784, cátedras 873, 1563, 341, 323, Red HAWC, México; DGAPA-UNAM grants IG101320, IN111716-3, IN111419, IA102019, IN110621, IN110521; VIEP-BUAP; PIFI 2012, 2013, PROFOCIE 2014, 2015; the University of Wisconsin Alumni Research Foundation; the Institute of Geophysics, Planetary Physics, and Signatures at Los Alamos National Laboratory; Polish Science Centre grant, DEC-2017/27/B/ST9/02272; Coordinación de la Investigación Científica 
de la Universidad Michoacana; Royal Society - Newton Advanced Fellowship 180385; Generalitat Valenciana, grant CIDEGENT/2018/034; Chulalongkorn University's CUniverse (CUAASC) grant; Coordinación General Académica e Innovación (CGAI-UdeG), PRODEP-SEP UDG-CA499; Institute of Cosmic Ray Research (ICRR), University of Tokyo, H.F. acknowledges support by NASA under award number 80GSFC21M0002. We also acknowledge the significant contributions over many years of Stefan Westerhoff, Gaurang Yodh and Arnulfo Zepeda Dominguez, all deceased members of the HAWC collaboration. Thanks to Scott Delay, Luciano Díaz and Eduardo Murrieta for technical support.

\section{References}

[1] A. Albert et al "3HWC: The Third HAWC Catalog of Very-high-energy Gamma-Ray Sources" 2020 ApJ 90576

[2] Lyne, Andrew \& Stappers, Ben \& Keith, Michael \& Ray, Paul \& Kerr, Mônica \& Camilo, Fernando \& Johnson, Tyrel. (2015). The binary nature of PSR J2032+4127. Monthly Notices of the Royal Astronomical Society. 451. 10.1093/mnras/stv236.

[3] A. U. Abeysekara et al, "Measurement of the Crab Nebula Spectrum Past $100 \mathrm{TeV}$ with HAWC" 2019 ApJ 881134

[4] Abeysekara, A.U., Albert, A., Alfaro, R. et al. "HAWC observations of the acceleration of very-high-energy cosmic rays in the Cygnus Cocoon." Nat Astron 5, 465-471 (2021). https://doi.org/10.1038/s41550-021-01318-y

[5] A. Albert et al, "Spectrum and Morphology of the Very-high-energy Source HAWC J2019+368" 2021 ApJ 911143

[6] E. Aliu et al, "OBSERVATIONS OF THE UNIDENTIFIED GAMMA-RAY SOURCE TeV J2032+4130 BY VERITAS" 2014 ApJ 78316

[7] Aharonian, F. et al. "The unidentified TeV source (TeV J2032+4130) and surrounding field" 224 Final HEGRA IACT-System results. Astron. Astrophys. 431, 197-202 (2005)

[8] Knödlseder, J. "On the massive star contents of Cygnus OB2", 2000, A\&A, 360, 539

[9] Hona, B. for the HAWC collaboration, PoS (ICRC 2019) 699

[10] Sinnis, G. "Water Cherenkov Detectors for Gamma-Ray Astronomy", World Scientific Review (2017)

[11] Vianello, G., et al. 2015, 34th ICRC, PoS, 1042

[12] Fleischhack, H. for the HAWC collaboration. PoS(ICRC2019)675

[13] Joshi, V. "Reconstruction and Analysis of Highest Energy gamma-rays and its Application to Pulsar Wind Nebulae", PhD Thesis, Ruperto-Carola University, Germany (2019) 
[14] Cao, Z., Aharonian, F.A., An, Q. et al. Ultrahigh-energy photons up to 1.4 petaelectronvolts from $12 \gamma$-ray Galactic sources. Nature 594, 33-36 (2021). https://doi.org/10.1038/s41586021-03498-z 


\section{Full Authors List: HAWC Collaboration}

A.U. Abeysekara ${ }^{48}$, A. Albert ${ }^{21}$, R. Alfaro ${ }^{14}$, C. Alvarez ${ }^{41}$, J.D. Álvarez ${ }^{40}$, J.R. Angeles Camacho ${ }^{14}$, J.C. Arteaga-Velázquez ${ }^{40}$, K. P. Arunbabu $^{17}$, D. Avila Rojas ${ }^{14}$, H.A. Ayala Solares ${ }^{28}$, R. Babu ${ }^{25}$, V. Baghmanyan ${ }^{15}$, A.S. Barber ${ }^{48}$, J. Becerra Gonzalez ${ }^{11}$, E. BelmontMoreno $^{14}$, S.Y. BenZvi ${ }^{29}$, D. Berley ${ }^{39}$, C. Brisbois ${ }^{39}$, K.S. Caballero-Mora ${ }^{41}$, T. Capistrán ${ }^{12}$, A. Carramiñana ${ }^{18}$, S. Casanova ${ }^{15}$, O. Chaparro-Amaro $^{3}$, U. Cotti ${ }^{40}$, J. Cotzomi ${ }^{8}$, S. Coutiño de León ${ }^{18}$, E. De la Fuente ${ }^{46}$, C. de León ${ }^{40}$, L. Diaz-Cruz ${ }^{8}$, R. Diaz Hernandez ${ }^{18}$, J.C. Díaz-Vélez ${ }^{46}$, B.L. Dingus ${ }^{21}$, M. Durocher ${ }^{21}$, M.A. DuVernois ${ }^{45}$, R.W. Ellsworth ${ }^{39}$, K. Engel ${ }^{39}$, C. Espinoza ${ }^{14}$, K.L. Fan ${ }^{39}$, K. Fang $^{45}$, M. Fernández Alonso ${ }^{28}$, B. Fick ${ }^{25}$, H. Fleischhack ${ }^{51,11,52}$, J.L. Flores ${ }^{46}$, N.I. Fraija ${ }^{12}$, D. Garcia ${ }^{4}$, J.A. García-González ${ }^{20}$, J. L. García-Luna ${ }^{46}$, G. García-Torales ${ }^{46}$, F. Garfias ${ }^{12}$, G. Giacinti ${ }^{22}$, H. Goksu ${ }^{22}$, M.M. González ${ }^{12}$, J.A. Goodman ${ }^{39}$, J.P. Harding ${ }^{21}$, S. Hernandez ${ }^{14}$, I. Herzog ${ }^{25}$, J. Hinton ${ }^{22}$, B. Hona ${ }^{48}$, D. Huang ${ }^{25}$, F. Hueyotl-Zahuantitla ${ }^{41}$, C.M. Hui ${ }^{23}$, B. Humensky ${ }^{39}$, P. Hüntemeyer ${ }^{25}$, A. Iriarte ${ }^{12}$, A. Jardin-Blicq ${ }^{22,49,50}$, H. Jhee ${ }^{43}$, V. Joshi ${ }^{7}$, D. Kieda ${ }^{48}$, G J. Kunde ${ }^{21}$, S. Kunwar ${ }^{22}$, A. Lara ${ }^{17}$, J. Lee ${ }^{43}$, W.H. Lee ${ }^{12}$, D. Lennarz ${ }^{9}$, H. León Vargas ${ }^{14}$, J. Linnemann ${ }^{24}$, A.L. Longinotti ${ }^{12}$, R. López-Coto ${ }^{19}$, G. Luis-Raya ${ }^{44}$, J. Lundeen ${ }^{24}$, K. Malone ${ }^{21}$, V. Marandon $^{22}$, O. Martinez ${ }^{8}$, I. Martinez-Castellanos ${ }^{39}$, H. Martínez-Huerta ${ }^{38}$, J. Martínez-Castro ${ }^{3}$, J.A.J. Matthews ${ }^{42}$, J. McEnery ${ }^{11}$, P. Miranda-Romagnoli $^{34}$, J.A. Morales-Soto ${ }^{40}$, E. Moreno ${ }^{8}$, M. Mostafá ${ }^{28}$, A. Nayerhoda ${ }^{15}$, L. Nellen ${ }^{13}$, M. Newbold ${ }^{48}$, M.U. Nisa ${ }^{24}$, R. Noriega-Papaqui ${ }^{34}$, L. Olivera-Nieto ${ }^{22}$, N. Omodei ${ }^{32}$, A. Peisker ${ }^{24}$, Y. Pérez Araujo ${ }^{12}$, E.G. Pérez-Pérez ${ }^{44}$, C.D. Rho ${ }^{43}$, C. Rivière ${ }^{39}$, D. Rosa-Gonzalez $^{18}$, E. Ruiz-Velasco ${ }^{22}$, J. Ryan ${ }^{26}$, H. Salazar ${ }^{8}$, F. Salesa Greus ${ }^{15,53}$, A. Sandoval ${ }^{14}$, M. Schneider ${ }^{39}$, H. Schoorlemmer ${ }^{22}$, J. Serna-Franco ${ }^{14}$, G. Sinnis ${ }^{21}$, A.J. Smith ${ }^{39}$, R.W. Springer ${ }^{48}$, P. Surajbali ${ }^{22}$, I. Taboada ${ }^{9}$, M. Tanner ${ }^{28}$, K. Tollefson ${ }^{24}$, I. Torres ${ }^{18}$, R. Torres-Escobedo $^{30}$, R. Turner ${ }^{25}$, F. Ureña-Mena ${ }^{18}$, L. Villaseñor ${ }^{8}$, X. Wang ${ }^{25}$, I.J. Watson ${ }^{43}$, T. Weisgarber ${ }^{45}$, F. Werner ${ }^{22}$, E. Willox ${ }^{39}$, J. Wood ${ }^{23}$, G.B. Yodh ${ }^{35}$, A. Zepeda ${ }^{4}$, H. Zhou ${ }^{30}$

${ }^{1}$ Barnard College, New York, NY, USA, ${ }^{2}$ Department of Chemistry and Physics, California University of Pennsylvania, California, PA, USA, ${ }^{3}$ Centro de Investigación en Computación, Instituto Politécnico Nacional, Ciudad de México, México, ${ }^{4}$ Physics Department, Centro de Investigación y de Estudios Avanzados del IPN, Ciudad de México, México, ${ }^{5}$ Colorado State University, Physics Dept., Fort Collins, CO, USA, ${ }^{6}$ DCI-UDG, Leon, Gto, México, ${ }^{7}$ Erlangen Centre for Astroparticle Physics, Friedrich Alexander Universität, Erlangen, BY, Germany, ${ }^{8}$ Facultad de Ciencias Físico Matemáticas, Benemérita Universidad Autónoma de Puebla, Puebla, México, ${ }^{9}$ School of Physics and Center for Relativistic Astrophysics, Georgia Institute of Technology, Atlanta, GA, USA, ${ }^{10}$ School of Physics Astronomy and Computational Sciences, George Mason University, Fairfax, VA, USA, ${ }^{11}$ NASA Goddard Space Flight Center, Greenbelt, MD, USA, ${ }^{12}$ Instituto de Astronomía, Universidad Nacional Autónoma de México, Ciudad de México, México, ${ }^{13}$ Instituto de Ciencias Nucleares, Universidad Nacional Autónoma de México, Ciudad de México, México, ${ }^{14}$ Instituto de Física, Universidad Nacional Autónoma de México, Ciudad de México, México, ${ }^{15}$ Institute of Nuclear Physics, Polish Academy of Sciences, Krakow, Poland, ${ }^{16}$ Instituto de Física de São Carlos, Universidade de São Paulo, São Carlos, SP, Brasil, ${ }^{17}$ Instituto de Geofísica, Universidad Nacional Autónoma de México, Ciudad de México, México, ${ }^{18}$ Instituto Nacional de Astrofísica, Óptica y Electrónica, Tonantzintla, Puebla, México, ${ }^{19}$ INFN Padova, Padova, Italy, ${ }^{20}$ Tecnologico de Monterrey, Escuela de Ingeniería y Ciencias, Ave. Eugenio Garza Sada 2501, Monterrey, N.L., 64849, México, ${ }^{21}$ Physics Division, Los Alamos National Laboratory, Los Alamos, NM, USA, ${ }^{22}$ Max-Planck Institute for Nuclear Physics, Heidelberg, Germany, ${ }^{23}$ NASA Marshall Space Flight Center, Astrophysics Office, Huntsville, AL, USA, ${ }^{24}$ Department of Physics and Astronomy, Michigan State University, East Lansing, MI, USA, ${ }^{25}$ Department of Physics, Michigan Technological University, Houghton, MI, USA, ${ }^{26}$ Space Science Center, University of New Hampshire, Durham, NH, USA, ${ }^{27}$ The Ohio State University at Lima, Lima, OH, USA, ${ }^{28}$ Department of Physics, Pennsylvania State University, University Park, PA, USA, ${ }^{29}$ Department of Physics and Astronomy, University of Rochester, Rochester, NY, USA, ${ }^{30}$ Tsung-Dao Lee Institute and School of Physics and Astronomy, Shanghai Jiao Tong University, Shanghai, China, ${ }^{31}$ Sungkyunkwan University, Gyeonggi, Rep. of Korea, ${ }^{32}$ Stanford University, Stanford, CA, USA, ${ }^{33}$ Department of Physics and Astronomy, University of Alabama, Tuscaloosa, AL, USA, ${ }^{34}$ Universidad Autónoma del Estado de Hidalgo, Pachuca, Hgo., México, ${ }^{35}$ Department of Physics and Astronomy, University of California, Irvine, Irvine, CA, USA, ${ }^{36}$ Santa Cruz Institute for Particle Physics, University of California, Santa Cruz, Santa Cruz, CA, USA, ${ }^{37}$ Universidad de Costa Rica, San José, Costa Rica, ${ }^{38}$ Department of Physics and Mathematics, Universidad de Monterrey, San Pedro Garza García, N.L., México, ${ }^{39}$ Department of Physics, University of Maryland, College Park, MD, USA, ${ }^{40}$ Instituto de Física y Matemáticas, Universidad Michoacana de San Nicolás de Hidalgo, Morelia, Michoacán, México, ${ }^{41}$ FCFM-MCTP, Universidad Autónoma de Chiapas, Tuxtla Gutiérrez, Chiapas, México, ${ }^{42}$ Department of Physics and Astronomy, University of New Mexico, Albuquerque, NM, USA, ${ }^{43}$ University of Seoul, Seoul, Rep. of Korea, ${ }^{44}$ Universidad Politécnica de Pachuca, Pachuca, Hgo, México, ${ }^{45}$ Department of Physics, University of Wisconsin-Madison, Madison, WI, USA, ${ }^{46}$ CUCEI, CUCEA, Universidad de Guadalajara, Guadalajara, Jalisco, México, ${ }^{47}$ Universität Würzburg, Institute for Theoretical Physics and Astrophysics, Würzburg, Germany, ${ }^{48}$ Department of Physics and Astronomy, University of Utah, Salt Lake City, UT, USA, ${ }^{49}$ Department of Physics, Faculty of Science, Chulalongkorn University, Pathumwan, Bangkok 10330, Thailand, ${ }^{50}$ National Astronomical Research Institute of Thailand (Public Organization), Don Kaeo, MaeRim, Chiang Mai 50180, Thailand, ${ }^{51}$ Department of Physics, Catholic University of America, Washington, DC, USA, ${ }^{52}$ Center for Research and Exploration in Space Science and Technology, NASA/GSFC, Greenbelt, MD, USA, ${ }^{53}$ Instituto de Física Corpuscular, CSIC, Universitat de València, Paterna, Valencia, Spain 